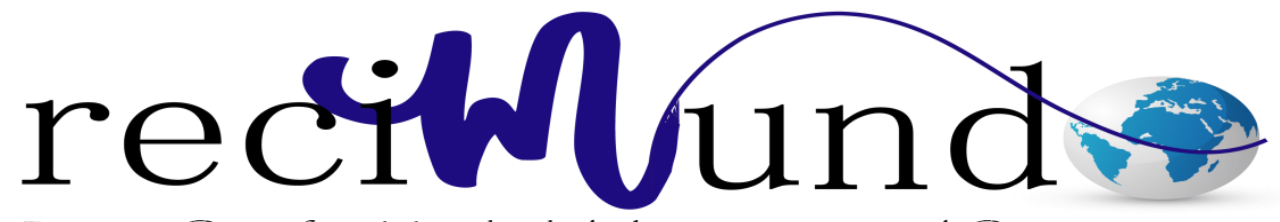

Revista Científica Mundo de la Investigación y el Conocimiento

Ing. Florencio Iván García Álvarez, MBA a ; Ing. Juan Bautista Solis Muñoz, MBA b; Ing. Xavier Augusto Mantilla Crespo, MBA ${ }^{\mathrm{c}}$

Impacto del turismo religioso en la ciudad de Azogues, Ecuador. Caso: Virgen de la Nube

Revista Científica Mundo de la Investigación y el Conocimiento. Vol. 2 núm., especial, mayo, ISSN: 2588-073X, 2018, pp. 525-559

DOI: 10.26820/recimundo/2.esp.2018.525-559

Editorial Saberes del Conocimiento

Recibido: $11 / 12 / 2017$

Aceptado: 01/02/2018

a. Universidad Católica de Cuenca Sede Azogues; figarciaa@ucacue.edu.ec

b. Universidad Católica de Cuenca Sede Azogues; jbsolizm@ucacue.edu.ec

c. Universidad Católica de Cuenca Sede Azogues; xamantillac@ucacue.edu.ec 


\section{Impacto del turismo religioso en la ciudad de Azogues, Ecuador. Caso: Virgen de la Nube}

Vol. 2, núm. Esp., (2018)

Ing. Florencio Iván García Álvarez, MBA; Ing. Juan Bautista Solis Muñoz, MBA; Ing. Xavier Augusto Mantilla Crespo, MBA

\section{RESUMEN}

El artículo tiene como objetivo explorar el impacto de las principales variables cuantitativas y cualitativas del turismo religioso en la ciudad de Azogues. Caso: Virgen de la Nube. Metodológicamente es de enfoque mixto, tipo exploratorio, probabilístico y de corte no experimental. La averiguación cuantitativa promueve al menos catorce variables medibles alrededor de tres factores de competitividad del destino. Los hallazgos con puntaje ponderado de 3, son las variables clave: Tamaño de turismo; frecuencia de turismo; y, canales de publicidad. La caracterización cualitativa de las categorías de análisis, muestra la necesitad de la implementación de planes de acción para posicionar, sustentar y rentabilizar el destino turístico en estudio, mejorando las percepciones negativas y potenciando las fortaleza del atractivo y los servicios. Así como la generación y potenciación de nuevas destinos turísticos anclados al producto estrella. ¿Es posible entonces cambiar las debilidades del destino turístico y potenciar el aprovechamiento de las oportunidades que genera el turismo religioso en Azogues? Queda demostrada la necesidad de crear la Gerencia de Gestión Turística en el organigrama de la Orden Franciscana. Las decisiones en manos de los hacedores de la política pública en los distintos niveles de gobierno y de la propia Comunidad Franciscana de Azogues.

Palabras claves: Turismo Religioso, Virgen de la Nube, Procesión del 1 de Enero, Azogues, Santuario Franciscano. 


\title{
Impacto del turismo religioso en la ciudad de Azogues, Ecuador. Caso: Virgen \\ de la Nube
}

Vol. 2, núm. Esp., (2018)

Ing. Florencio Iván García Álvarez, MBA; Ing. Juan Bautista Solis Muñoz, MBA; Ing. Xavier Augusto Mantilla Crespo, MBA

\begin{abstract}
The aim of this article is to explore the impact of the main quantitative and qualitative variables of religious tourism in Azogues city. Case: Virgen de la Nube. The methodology it is a combination of the exploratory, probabilistic and non-experimental approaches. The quantitative inquiry promotes at least fourteen measurable variables around three factors of competitiveness of the destination. The findings with a weighted score of 3 are the key variables like tourism size, frequency of tourism, and advertising channels. The qualitative characterization of the categories of analysis shows the need for the implementation of action plans to position, sustain and profitability of the tourist destination under study, it requires to improve the negative perceptions and enhance the strength of attractiveness and services. As well as the generation and promotion of new tourist destinations anchored to the star product. Is it possible to change the weaknesses of the tourist destination and enhance the use of the opportunities generated by religious tourism in Azogues? The need to create the Department of Tourism Management in the organigram of the Franciscan Order is demonstrated. The decisions are in hands of the public policy makers at the different levels of government and the Franciscan Community of Azogues itself.
\end{abstract}

Keywords: Religious Tourism, Virgin of the Cloud, Procession of January 1, Azogues, Franciscan Sanctuary. 


\section{Impacto del turismo religioso en la ciudad de Azogues, Ecuador. Caso: Virgen de la Nube}

Vol. 2, núm. Esp., (2018)

Ing. Florencio Iván García Álvarez, MBA; Ing. Juan Bautista Solis Muñoz, MBA; Ing. Xavier Augusto Mantilla Crespo, MBA

\section{Introducción.}

La línea teórica se amolda a rastrear los aportes de investigadores inquietos por saber los entretelones del turismo religioso, desde los conceptos asociados: La fe y religiosidad popular que en opinión de Arias (2018) la gente llega a los templos preferentemente a rezar y escasamente a reflexionar y aprender del lenguaje abordado desde lo icónico; y, los signos gestuales y símbolos, traducidos en lenguaje iconográfico de los destinos religiosos, en conexión e importancia hacia la incidencia en las variables de identidad cultural y socioeconómicas. La producción de literatura religiosa dedicada el primer concepto es virtuosa; sin embargo, hay una tarea pendiente en el ámbito de la investigación del turismo religioso en todo el Ecuador, sobre la línea del lenguaje visual expresado en el arte: Qué significa el rosetón, el triforio, la indumentaria de la Virgen, el dogma de la Inmaculada. Qué implicación tiene la nave del evangelio, el retablo, el púlpito, el presbiterio, la luz natural como penetración de Dios en el alma, las vidrieras, los edificios románicos, el arco de medio punto en los templos góticos, las imágenes de candelero o policromadas que en apreciación de (Arias, Op. Cit., 2018) son libros visuales de la historia del turismo religioso. Estos que en general están en blanco, sus páginas están casi vacías. Así, por ejemplo, para Gabriela Eljuri, el enfoque de la Fiesta de la Virgen de la Nube en Nueva York merece más bien un análisis de tipo antropológico, basada en la publicación de la UPS (2003) sobre Migrantes de Azuay y Cañar en la Gran Nueva York, Cultura e Identidad, al referir a esta última como un proceso dinámico y en construcción. El ángulo ecológico también tiene cabida en peregrinaciones religiosas multitudinarias como propone Armijos (2015) en la investigación: Plan de concientización ambiental en la romería del Quinche, en la provincia de Pichincha, trabajo que se basa en que los dos millones de peregrinos que caminarán hasta la Basílica de los Angeles 


\section{Impacto del turismo religioso en la ciudad de Azogues, Ecuador. Caso: Virgen \\ de la Nube}

Vol. 2, núm. Esp., (2018)

Ing. Florencio Iván García Álvarez, MBA; Ing. Juan Bautista Solis Muñoz, MBA; Ing. Xavier Augusto Mantilla Crespo, MBA

(2011), llevarán consigo una promesa más: Manejar correctamente los residuos y disponerlos de

forma adecuada para su reciclaje. Lourdes y Fátima, son otro ejemplo de orden. El camino de

Santiago está provisto de albergues, donde la incidencia económica en el área de influencia es notoria al elevar el nivel de ingreso en forma sostenida durante todo el año. Más parece que los destinos turísticos religiosos en el Ecuador están condenados a levantarse en puntuales épocas del año.

En la búsqueda del encuadre espacio temporal sobre la fe y la religiosidad mariana, para Cavalo (1964) citado por Toledo (2007) la realización de las fiestas religiosas en los pueblos amerindios, se da a partir de la Bula de Paulo III, difundida por el Concilio de Lima (...) están reservados los Indios de guardar otras fiestas a más de los domingos del año: Navidad, Resurrección, Venida del Espíritu Santo, Fiesta de los Reyes, día de la Ascensión, Corpus Christi, Fiesta de Nuestra Señora, Natividad, Purificación, Anunciación, Asunción, apóstoles Pedro y Pablo y no otro día alguno. Esta orden se consideraba privilegio y facultades concedidas para los Indios. Según Toledo (Op. Cit) en Fiestas Populares una Alternativa del Turismo Cultural en la Provincia del Cañar (p. 81) la Virgen de la Nube es muy venerada en el Austro particularmente en la ciudad de Azogues, refiriéndose a las dos procesiones anuales, la del 1 de enero y la del 31 de mayo, señala: Las calles por donde pasa la Virgen son adornadas con globos y demás ornamentas de vistosos colores. Luego del recorrido de la procesión se llega a la iglesia donde la imagen es depositada en el altar principal (...) y luego de corear el Himno Nacional finaliza la fiesta del uno de Enero de cada año. 


\section{Impacto del turismo religioso en la ciudad de Azogues, Ecuador. Caso: Virgen}

de la Nube

Vol. 2, núm. Esp., (2018)

Ing. Florencio Iván García Álvarez, MBA; Ing. Juan Bautista Solis Muñoz, MBA; Ing. Xavier Augusto Mantilla Crespo, MBA

Desde la producción académica de Tobay (2008) el turismo religioso es un tipo de turismo muy conocido y practicado en todo el mundo. En Ecuador es una de las actividades más conocidas (...) especialmente en la ciudad de Loja, donde el turismo religioso se lo viene desarrollando desde hace por lo menos 400 años. Para Rubio \& Mariscal (2005 citado por Ulloa (2009) turismo religioso son los flujos de viajeros cuya principal motivación es la concurrencia a centros receptivos de carácter religioso, el fenómeno excede de las características que como producto de ocio entraña cualquier destino turístico, así como a la mera atribución que corresponde a los peregrinos como consumidores. Fortaleciendo la definición, las potencialidades que agrega el turismo religioso tienen virtudes exponenciales.

López de Atienza (1583) cuando se refiere a las relaciones de la Audiencia de Quito, anota las doctrinas que hay en términos de la ciudad de Cuenca, de Clérigos y Religiosos de San Francisco, tienen las doctrinas de Paute, Gualaceo y Molleturo. Los agustinos no tienen en el distrito ninguna doctrina. Los clérigos tienen las siguientes doctrinas: (...) La doctrina El Azogue en Gaspar de Gallegos.

Según Ramírez (1998) en Cañar, Provincia Emérita, la provincia del Cañar fue erigida como tal, el 3 de noviembre de 1880. El 17 de noviembre, el ejecutivo puso el ejecútese. Se creó como provincia de Azogues, con su capital Azogues. Hacia 1884 con la expedición de la Ley de División Territorial queda nominada como provincia del Cañar, con su capital Azogues. Desde los albores de la provincia, Azogues fue servida por los religiosos Oblatos (1897). Hay evidencia de la permanencia de sacerdotes oblatos en Azogues como Virgilio Maldonado y Manuel Ordóñez Alvarez, quien quiso fundar una capilla para la veneración de San José. Consultó a su prelado en 


\section{Impacto del turismo religioso en la ciudad de Azogues, Ecuador. Caso: Virgen \\ de la Nube}

Vol. 2, núm. Esp., (2018)

Ing. Florencio Iván García Álvarez, MBA; Ing. Juan Bautista Solis Muñoz, MBA; Ing. Xavier Augusto Mantilla Crespo, MBA

Cuenca, Julio María Matovelle, autoridad eclesiástica que replicó: "Pero no será esa capilla para

San José, sino para la Virgen de la Nube”.

Sobre la unidad de análisis: la Virgen de la Nube; el Santuario Nacional, regentado por los padres franciscanos en la ciudad de Azogues; la procesión religiosa de cada 1 de enero en el contexto de la fe y religiosidad popular, evidentemente se sustenta en el hecho histórico de la aparición registrado el 30 de diciembre de 1696 en la ciudad de Quito. Datos históricos encontrados en la Biblioteca Nacional Eugenio Espejo, rastreados por el investigador Orellana (1948) revelan que en efecto la población quiteña vivió momento de angustia por la enfermedad de su obispo Sancho de Andrade y Figueroa. La ciudadanía se puso de pie y organizó una procesión de rogativa por la salud del Prelado. La procesión recorrió las calles de Quito. Arribó al pretil del Templo Franciscano. Sonó la campanilla, eran las cuatro y cuarenta y cinco de la tarde, la feligresía de rodillas. El ambiente fue perturbado por las voces del Presbítero José de Ulloa y la Cadena, quien al alzar la mirada al firmamento, contempló en el cielo a la Virgen. El proceso canónico de la aparición de la Virgen se hizo en Quito bajo la dirección del Vicario General de la Diócesis Pedro de Zumárraga. Los documentos probatorios fueron publicados en Cuenca por el padre Julio María Matovelle, en 1900. Poco después de la aparición de María, se adjetivó como Nuestra Señora de la Nube. Según Orellana (1948) en la misma ciudad de Quito, no se ha notado ferviente devoción por la Virgen de la Nube. Más allá de las celebraciones del primero y segundo centenario de la Aparición, no se ha decidido levantar un templo que perpetúe la memoria del beneficio de María.

Con el criterio de Matovelle, el sacerdote Manuel Ordóñez Alvarez, buscó el sitio propicio para levantar la capilla. Al noroeste de Azogues se levanta un enorme anfiteatro de montañas. 


\section{Impacto del turismo religioso en la ciudad de Azogues, Ecuador. Caso: Virgen}

de la Nube

Vol. 2, núm. Esp., (2018)

Ing. Florencio Iván García Álvarez, MBA; Ing. Juan Bautista Solis Muñoz, MBA; Ing. Xavier Augusto Mantilla Crespo, MBA

Siendo la colina denominada "El Calvario" donde se erigió la capilla a la Virgen de la Nube. El

pueblo fue invitado para la colocación de la primera piedra, el 29 de agosto de 1897, que fue bendecida por el Administrador Apostólico de la Diócesis de Cuenca, Benigno Palacios. Así se estableció el culto a la Virgen de la Nube en Azogues, con una imagen trabajada por el artista Daniel Alvarado, de Cuenca.

Siguiendo la postura argumentativa de Orellana (1948) en atención a las gestiones del Obispo de Cuenca, Manuel María Pólit Laso, el padre provincial de los franciscanos sacerdote José María Aguirre, consiguió para que la orden franciscana viniera Azogues, el 21 de febrero de 1912 proveniente de Quito, para la nueva fundación. Lo hicieron los frailes Santiago Gómez y José María Idígoras, hospedándose en la casa parroquial, siendo párroco Froilán Pozo Quevedo. Se escogió el lugar del nuevo convento la montaña del Calvario, junto a la capilla de la Virgen de la Nube, el 8 de diciembre de 1912.

Desde un principio se vio la necesidad de edificar un templo. El fraile franciscano José María Idígoras, instaló el 28 de abril de 1912, el sistema de mingas. Se colectó gran cantidad de material para la construcción. Según la Revista Nomenclaturas (2014) fue Froilán Pozo Quevedo, el religioso que donó los terrenos donde se edificó el templo. Este era completamente rocoso, hasta el 13 de enero de 1917, se desalojó las piedras del área de construcción, hasta colocar los cimientos de cuatro metros de profundidad. El sacerdote Saturdino Gorri continuó la edificación y el 26 de mayo de 1927, se bendijo el presbiterio del Santuario. En la primera guardianía del fraile franciscano Rufino Urtaza, se concluyó con el entablado del Santuario, construyéndose la torre, en 1942. Instalando el reloj público, adquirido en 1922 de fabricación alemana. La longitud del 


\section{Impacto del turismo religioso en la ciudad de Azogues, Ecuador. Caso: Virgen \\ de la Nube}

Vol. 2, núm. Esp., (2018)

Ing. Florencio Iván García Álvarez, MBA; Ing. Juan Bautista Solis Muñoz, MBA; Ing. Xavier Augusto Mantilla Crespo, MBA

templo es de cuarenta metros y su latitud de dieciocho. La dirección de la obra lo hizo el sacerdote

Hilario González. Se construyó a la par el pretil iniciado en 1944 y concluido hacía 1947. Según

el índice biográfico de artistas plásticos del Cañar, al hablar de Daniel Mogrovejo Narváez, sostiene que la comunidad franciscana proyectaba un nuevo templo dedicado a la advocación de la Virgen de la Nube. Fue el momento para que Mogrovejo sea llamado a colaborar en los dibujos y parte de los diseños de la fachada del templo, interiores y antiguos altares. En el centro superior del Santuario, está la imagen de la Virgen, tiene tres metros de altura, en tres piezas de mármol traído de Zhirincay, elaborada por el escultor Mogrovejo Narváez, discípulo de Daniel Alvarado Bermeo (Domínguez, 2006, p. 324). Continuando en la línea de los aportes de Domínguez (2006) al hablar de la imagen de la Virgen de la Nube, le atribuye al escultor cuencano Daniel Salvador Alvarado, obra realizada en 1896, así consta del propio testimonio del escultor, doy fe y testimonio que el Padre Julio María Matovelle, fundador de los Oblatos, me contrató para que trabajase la imagen de la Santísima Virgen de la Nube, que hoy posee el Convento- Santuario de los padres franciscanos de Azogues.

Se ha encontrado entre las opiniones de los historiadores que, Julio María Matovelle, sacerdote perteneciente a la congregación de oblatos, hizo pintar en Quito como favor por un milagro recibido de la Virgen de la Nube a un religioso de la Orden Oblata, un cuadro de la Aparición, con el pintor Joaquin Pinto. La obra pictórica fue obsequiada por Matovelle al Convento de Azogues, el 8 de diciembre de 1920. Cabe anotar que según el rastreo del estado del arte, en Azogues está el único Santuario donde se venera la Virgen de la Nube, regentado por los frailes franciscanos. 


\section{Impacto del turismo religioso en la ciudad de Azogues, Ecuador. Caso: Virgen}

de la Nube

Vol. 2, núm. Esp., (2018)

Ing. Florencio Iván García Álvarez, MBA; Ing. Juan Bautista Solis Muñoz, MBA; Ing. Xavier Augusto Mantilla Crespo, MBA

Según Matovelle (1900) en Nuestra Señora de la Nube, la labor espiritual de los franciscanos en

Azogues, ha sido múltiple. El número de romeros cada uno de enero llega a los veinte mil que acuden a la fiesta y procesión. Acuden también los ciento veinte coros fundados en agosto de 1922.

Funcionan, en el Santuario, las siguientes congregaciones: La Tercera Orden; la Congregación Primaria de Nuestra Señora de la Nube; la del Sagrado Corazón de Jesús y de la Beata Mariana; Terciaria Franciscana. En el año 1947 se vivió las bodas de oro del establecimiento del culto a la Virgen en Azogues (1897). La Virgen de la Nube es salud de los enfermos, desde que sanó al Obispo Figueroa, se la invoca: Dios te salve, salud de los enfermos.

La coronación canónica de la Santísima Virgen de la Nube, tal como se recuerda en la publicación de Díaz (1967) fue un fausto evento que acrecentó en las almas el amor mariano. Siendo su santidad el papa Paulo VI quien concedió el 24 de octubre de 1965, el Decreto de Coronación Canónica a la Virgen de la Nube. Monseñor Manuel de Jesús Serrano Abad, Arzobispo de la Arquidiócesis de Cuenca, en calidad de delegado pontificio, hizo la coronación canónica el 1 de enero de 1967.

Para Carrasco (2016) la Virgen de la Nube es venerada por los fieles locales y de todo el país y turistas, en el Santurario de Azogues. Sobre la ciudad el investigador añade que fue cantonizada el 16 de abril de 1825 y proclamó la independencia el 4 de noviembre de 1820. Según el Plan de Desarrollo y Ordenamiento Territorial Provincial Actualizado (2015) Azogues tiene un área urbana de 65,46 kilómetros cuadrados. Rastreado el Plan de Desarrollo Urbano de Azogues (1988) no existe evidencia dentro del patrimonio cultural edificado de una mención en la zona de planificación de San Francisco destinado a la preservación del Santurario Franciscano y su 


\section{Impacto del turismo religioso en la ciudad de Azogues, Ecuador. Caso: Virgen \\ de la Nube}

Vol. 2, núm. Esp., (2018)

Ing. Florencio Iván García Álvarez, MBA; Ing. Juan Bautista Solis Muñoz, MBA; Ing. Xavier Augusto Mantilla Crespo, MBA

entorno. Los planes de desarrollo y ordenamiento territorial como: Azogues, 2015, formulado en

el 2008 y que no tuvo rigor normativo por desavenencias políticas, como el PDOT del año 2013

que igualmente tuvo serios reveses en la etapa de aprobación, no desarrollan, sin embargo, planes,

programas, proyectos y actividades para potenciar el turismo religioso como fuente de oportunidades y generación de empleo productivo.

A su vez, desde el ángulo del Plan Nacional del Buen Vivir (2013-2017) se establece en el objetivo 8, consolidar el sistema económico social y solidario de forma sostenible. La nueva concepción apostaba a concretar aspectos como la inclusión económica. En esta línea, el objetivo 10, impulsar la transformación de la matriz productiva, en la orientación de nuevas industrias y promoción de nuevos sectores de alta productividad, competitiva, sostenible, sustentable y diversos, con visión territorial y de inclusión económica en los encadenamientos que generen. El Plan Nacional del Buen Vivir (2017- 2021) en el eje 2 de la economía al servicio de la sociedad, destina el objetivo 4 a consolidar la sostenibilidad del sistema económico social y solidario y afianzar la dolarización. El objetivo 5, expresa impulsar la productividad y competitividad para el crecimiento económico sustentable de manera redistributiva y solidaria. Finalmente, el objetivo 6, menciona desarrollar las capacidades productivas y del entorno para lograr la soberanía alimentaria (...). En aportes de la ciudadanía Tabla No. 3 en economía al servicio de la sociedad, se menciona el desarrollo de emprendimientos de turismo locales.

En la ruta de explorar los atractivos turísticos que posiblemente estén atados al producto estrella -la Virgen de la Nube-, el estudio se interesa a continuación sobre las principales ventajas comparativas y competitivas del Producto turístico: atractivos más servicios, que pueden 


\section{Impacto del turismo religioso en la ciudad de Azogues, Ecuador. Caso: Virgen}

de la Nube

Vol. 2, núm. Esp., (2018)

Ing. Florencio Iván García Álvarez, MBA; Ing. Juan Bautista Solis Muñoz, MBA; Ing. Xavier Augusto Mantilla Crespo, MBA

desarrollar ideas de emprendimiento. En el entorno competitivo del área de influencia del

Santuario, se destacan atractivos turísticos como el monumento a la Virgen de la Nube en la cima

del Abuga. El complejo de aguas termales en la parroquia Guapán. El complejo turístico de

Cojitambo. Los museos de la ciudad. En efecto, de acuerdo con los datos de la revista CulturisArte,

(2017) un fraile franciscano de nombre Manuel García, desde el visor de la ventana divisó una nube eterna incrustada en la montaña. Vino entonces la idea: Erigir una Virgen en lo más alto del centinela oriental. El monumento tiene una base de 5 metros de altura, calculado para una presión del viento de 300 kilómetros/ hora. Mide 25 metros de altura y pesa 45 toneladas. Los sistemas de anclaje, suelda y radiografía se sometieron a un proceso de control de calidad riguroso. La construcción es $100 \%$ mano de obra ecuatoriana, tanto en el intelecto cuanto en el esfuerzo motriz de quienes dieron forma a la agraciada imagen que bendice todos los días a la ciudad de Azogues. El atractivo se sitúa a los 3.100 metros sobre el nivel del mar. Se conoce de fuentes primarias que la obra impulsada por la Fundación Virgen de la Nube supera tensiones de grupos sociales conflictivos, gracias a la capacidad conciliadora y de liderazgo de Marco Vicuña Domínguez.

De acuerdo con los datos proporcionados por Vicuña (2016), con una inversión que sobrepasa los dos millones de dólares, el gobierno autónomo descentralizado provincial del Cañar, construye el Complejo Integral de Aguas Termales en la parroquia rural de Guapán. El complejo turístico de aguas termales Guapán, consta de los siguientes servicios privados como un conjunto de productos recreativos, terapéuticos, conformados por piscinas, SPA (Baños turcos, masajes y tratamientos), bar/restaurante, hotel, salón de eventos, comercio de artesanías, comercio de recuerdos. Como parte de los elementos públicos constan: Museo, templo, recorrido histórico, parque botánico, juegos de agua, escalada deportiva, pista de estilo libre, mirador, caminerías, 


\section{Impacto del turismo religioso en la ciudad de Azogues, Ecuador. Caso: Virgen \\ de la Nube}

Vol. 2, núm. Esp., (2018)

Ing. Florencio Iván García Álvarez, MBA; Ing. Juan Bautista Solis Muñoz, MBA; Ing. Xavier Augusto Mantilla Crespo, MBA

plaza multiuso, parqueaderos. Los elementos privados y públicos están emplazados en las 18.3

hectáreas del predio de la Junta Parroquial de Guapán.

La Serie Patrimonial, publicación de la Casa de la Cultura Núcleo del Cañar, (2010) señala las estructuras arquitectónicas de Cojitambo que están constituidas por aparejos de tipo rústico. El destino turístico de Cojitambo se ubica hacia la parte occidental del pueblo de Azogues, Carrillo (2010) y quiere decir asiento de holgura y descanso. El Ministerio del Deporte del Ecuador, instaló setenta puntos de escalada deportiva aprovechando la formación rocosa de andesita, Rojas (2005). En el 2007 el gobierno autónomo descentralizado parroquia rural de Cojitambo, asumió la celebración de la Fiesta del Maíz, coincidente con el solsticio de junio. Para el 2014 el gobierno autónomo descentralizado municipal de Azogues, dictó la Ordenanza que norma la Fiesta del Maíz en Cojitambo. El complejo paisajístico de altura es visitado por turistas del país y del extranjero según un estudio exploratorio de la Universidad Católica de Cuenca Sede Azogues (2008) denominado Estudio de Diagnóstico del Segundo Festival del Maíz, Cojitambo 2008, dirigido a visitantes y participantes del festival (alrededor de 2910 personas) y 13 vendedores minoristas.

Según la ficha museográfica de la Casa de la Cultura Núcleo del Cañar, al hablar del museo etnográfico, se señala que los fondos del museo están integrados por 302 piezas de cerámica, piedra, madera, cuero, textiles, cestería, paja hojalatada, adquiridos directamente en las comunidades indígenas y mestizas de la provincia del Cañar. Contiene una colección de 350 piezas arqueológicas de las culturas Narrío, Cañari, e Inca. A su vez, el sitio web: el ecuatoriano.com/ec, el museo del colegio Juan Bautista Vázquez, rescata la imprenta de gobierno junto a otros materiales museográficos, posee material bibliográfico que van desde el año 1553, escritas en latín 


\section{Impacto del turismo religioso en la ciudad de Azogues, Ecuador. Caso: Virgen de la Nube}

Vol. 2, núm. Esp., (2018)

Ing. Florencio Iván García Álvarez, MBA; Ing. Juan Bautista Solis Muñoz, MBA; Ing. Xavier Augusto Mantilla Crespo, MBA

y traducidas en Romance Castellano, otras calificadas como incunables por su valor filosófico,

literario e histórico. No existen levantadas las fichas del museo del Convento Franciscano, como tampoco del museo del Colegio La Salle.

Pero, la devoción a la Virgen de la Nube, va más allá de Azogues y Quito, hace rato que trascendió las fronteras patrias para situarse en los Estados Unidos y Canadá. En la línea del rastreo documental encontramos la investigación realizada por Gabriela Eljuri (Op. Cit.), en la Virgen de la Nube en Manhattan, la religiosidad popular más allá de la frontera (p.18) en las provincias de Azuay y Cañar el culto a esta Virgen es muy importante, especialmente, en los sectores populares. Cada año los feligreses acuden a la capilla de la Virgen de la Nube en Azogues en demostración de su devoción. En los Estados Unidos los migrantes de estas dos provincias no han dejado su fe hacia su patrona y al contrario la mantienen incluso más viva que antes. De la lectura hecha a Un Siglo de Fe y Prodigios, edición conmemorativa centenaria, por los cien años de la presencia franciscana en Azogues, el investigador Donoso, (2012) al referirse a la Madre del Ecuatoriano Ausente, habla del culto de la Virgen de la Nube en New York y Norte América, enfatiza que este hecho comenzó en la primavera de 1988, cuando una familia Alvarado proveniente de Cuenca, afligida por el abandono de su hijo de nombre Juan, no retornaba al hogar. El párroco de Santa Ana, un sacerdote norteamericano solicitó a la familia una Virgen Ecuatoriana para celebrar una misa y pedir para que obre el milagro del retorno del niño extraviado. Los padres entregaron la imagen de la Virgen de la Nube. Desde esta vez, se formaron los primeros coros de la Virgen de la Nube. El 1 de enero de todos los años se celebra la misa y procesión en honor a la Virgen en el templo del Santo Rosario, en el Alto Manhattan. La fe pronto se propagó a Queens, Brooklyn, Pensilvania, Chicago, Toronto. Desde 1989 un grupo de azogueños, venía celebrando misas a la 


\section{Impacto del turismo religioso en la ciudad de Azogues, Ecuador. Caso: Virgen \\ de la Nube}

Vol. 2, núm. Esp., (2018)

Ing. Florencio Iván García Álvarez, MBA; Ing. Juan Bautista Solis Muñoz, MBA; Ing. Xavier Augusto Mantilla Crespo, MBA

Virgen de la Nube en el templo de San Bartolomé de Elmhurst Queens. Posteriormente se entronizó su imagen en la Catedral de San Patricio, la más importante de los Estados Unidos. Condo (2012)

\section{Importancia del Problema.}

El impacto del turismo religioso en la ciudad de Azogues, Ecuador. Caso: Virgen de la Nube, lo asumimos desde el perfil de los conceptos: Fe y religiosidad popular; y, lenguaje iconográfico. Dimensiones atadas claramente a las variables socio económicas y de identidad cultural en el contexto del posicionamiento, sostenibilidad y rentabilidad del turismo religioso visto desde la mirada de la unidad de análisis, la Virgen de la Nube y comparada con lo que pasa alrededor de la devoción a la Virgen del Cisne que se venera en Loja. De hecho, el turismo religioso alrededor del mundo tiene alto impacto en diferentes aristas. Visto desde la perspectiva de la Iglesia Católica, la fe y la religiosidad entendida como el culto popular son elementos que potencian la variable cultural del pueblo universal y particularmente de la comunidad latinoamericana. Su implicación en la sociedad es tal que los gobiernos en sus distintos niveles han generado, adecuado y revisado la política pública. Recuperando lo dicho en el apartado introductorio, la devoción a la Virgen de la Nube, ha trascendido fronteras y se ha posicionado mediante entronización en los principales estados de Norte América y Canadá. Lo que naturalmente nos lleva a pensar en la implícita aceptación de los gobiernos extranjeros a la manifestación religiosa profesada por miles de ecuatorianos y latinoamericanos. Desde el contexto económico, se plantea claramente el problema en la relación causal de si el destino turístico, es decir, atractivo más servicios alrededor del Santuario de la Virgen de la Nube, en la ciudad de 


\section{Impacto del turismo religioso en la ciudad de Azogues, Ecuador. Caso: Virgen}

de la Nube

Vol. 2, núm. Esp., (2018)

Ing. Florencio Iván García Álvarez, MBA; Ing. Juan Bautista Solis Muñoz, MBA; Ing. Xavier Augusto Mantilla Crespo, MBA

Azogues, así como sus manifestaciones de ejercicios religiosos y procesión del 1 de enero,

repercute y en qué medida potencia la economía en toda la cadena de los actores de las Mipymes,

en el importante sector de la economía popular y solidaria, contenida en el eje 2 del Plan Nacional del Buen Vivir (2017-2021). Se apunta a conocer las percepciones de los turistas nacionales y extranjeros en relación al estudio multivariable y caracterización de testimonios recogidos en instrumentos metodológicamente pre establecidos. Tomar el pulso a la ciudad relacionándole con el turismo religioso y registrar sus signos vitales, es esencial en la voz de la Academia. Se intenta decir, cuáles son las fortalezas y las debilidades corregibles desde las instancias decisionales. Se tiene como propósito identificar las amenazas y por cierto, explorar las reales oportunidades derivadas de la manifestación de fe que se traduce en un posicionado producto de turismo religioso. Es posible entonces, desarrollar encadenamientos de servicios turísticos. Se da por factible un enfoque mayormente sistémico, fuertemente articulado a los actores turísticos, prestadores de servicios, operadoras de turismo, rectores y generadores de la política pública, rol de las universidades en las líneas de investigación fuertemente atadas a los planes de desarrollo y ordenamiento territorial, agendas zonales, enfoques intersectoriales en función de los ejes, objetivos, políticas, programas y actividades del Plan Nacional del Buen Vivir. Finalmente mirar a profundidad si en aquel perfil de dos anclas, está o no desarrollado el lenguaje iconográfico del destino turístico desde una estrategia didáctica, pedagógica, educativa que sostenga en el tiempo las cuestiones del destino turístico. Esas implicaciones amarran la presente investigación bajo la rigurosidad del enfoque metodológico y la presente justificación del problema. 


\section{Impacto del turismo religioso en la ciudad de Azogues, Ecuador. Caso: Virgen \\ de la Nube}

Vol. 2, núm. Esp., (2018)

Ing. Florencio Iván García Álvarez, MBA; Ing. Juan Bautista Solis Muñoz, MBA; Ing. Xavier Augusto Mantilla Crespo, MBA

\section{Metodología.}

El presente artículo se enmarca metodológicamente en un estudio de enfoque mixto - con mediciones cuantitativas y aproximaciones cualitativas-, de tipo exploratorio, probabilístico y de corte no experimental. Al volver sobre los escritos de Roberto Hernández Sampieri (2008) el hilo conductor nos aproxima a la idea que en el estudio nace de la observación directa y la lectura relacionada a cómo impacta el factor religioso en la actividad dinámica del turismo interno y externo. Caso: Virgen de la Nube. La construcción del marco histórico y teórico responde al rastreo del estado de la cuestión. Al ser un trabajo con enfoque cuantitativo, sobre un universo de 20 mil potenciales turistas, usando el método aleatorio simple o irrestricto aleatorio se ha determinado el tamaño de la muestra con 2 desviaciones estándar, 95\% de nivel de confianza y +-5 de límite de error de estimación. El tamaño de la muestra "n" es de 392 encuestas. El levantamiento de la información de campo se ha tomado en cuatro días consecutivos a la procesión del 1 de enero de 2018 en el área de influencia del templo de San Francisco. Se han tabulado los datos y se ha analizado la información agrupada en variables de intervención, utilizando las herramientas SPSS y Atlas.Ti. Se han recopilado testimonios textuales de turistas en entrevistas no estructuradas para corroborar los hallazgos cuantitativos. Mediante la técnica de grupos focales con los actores de la economía popular y solidaria de Azogues, se han recogido los testimonios de los sectores: gastronomía, trasporte, hoteles y hostales, artesanías. No existen evidencias teóricas de trabajos previos al caso de análisis pero si se ha encontrado estudios extrapolables de la Virgen del Cisne, con el título: Impacto del factor religioso de la comunidad ecuatoriana en Madrid. El caso de la Virgen del Cisne. Se ha extrapolado mediante visita in situ a la Virgen del Cisne, donde se ha levantado información relevante sobre fe y religiosidad popular; y, lenguaje icónico desde los 


\section{Impacto del turismo religioso en la ciudad de Azogues, Ecuador. Caso: Virgen}

de la Nube

Vol. 2, núm. Esp., (2018)

Ing. Florencio Iván García Álvarez, MBA; Ing. Juan Bautista Solis Muñoz, MBA; Ing. Xavier Augusto Mantilla Crespo, MBA

signos gestuales y símbolos aplicados al caso de la Virgen del Cisne en un estudio de caso

financiado por la empresa $\mathrm{H} \& \mathrm{H}$. Con fundamento en la metodología que tiene un alto componente

de observación directa y toma de datos en campo, se sitúan las siguientes variables cuantitativas de rigor investigativo: V1. Procedencia; V2. Hábitos de Turismo; V3. Tamaño de turismo; V4. Frecuencia de Turismo; V5. Canales de Publicidad; V6. Medios de Transporte; V7. Señalética Turística; V8. Tiempo de permanencia en el destino; V9. Equipamientos de alojamiento; V10. Percepción del entorno natural y limpieza del destino; V11. Asepsia y sabor de la gastronomía del destino; V12. Seguridad del destino turístico; V13. Consumo en dólares; V14. Potenciales atractivos encadenados. Se construye la matriz que contiene las variables clave en función de los factores de competitividad: posicionamiento, sostenibilidad y rentabilidad, por el método de ponderación por puntos. Para la socialización de resultados se prevé comunicar mediante conferencia de prensa con los actores involucrados, ponencia académica y publicación en base de datos regional.

\section{Resultados.}

Desde el enfoque cuantitativo declarado en el renglón metodológico y que tiene como propósito medir la incidencia en la economía popular y solidaria del producto turístico: Virgen de la Nube, con sus derivaciones como la procesión del 1 de enero, el Santurario Franciscano como atractivo turístico, el corredor San Francisco - Señor de Flores- Virgen de la Nube en el Abuga. En esta línea numérica, porcentual, los hallazgos tienen el siguiente rigor: El 67\% de turistas que acuden a la Virgen de la Nube, entre el viernes 29 de diciembre de 2017 y lunes 1 de enero de 2018, proceden de la Sierra. El 14\% de la región costanera. El 18\% de los cantones de la provincia 


\section{Impacto del turismo religioso en la ciudad de Azogues, Ecuador. Caso: Virgen \\ de la Nube}

Vol. 2, núm. Esp., (2018)

Ing. Florencio Iván García Álvarez, MBA; Ing. Juan Bautista Solis Muñoz, MBA; Ing. Xavier Augusto Mantilla Crespo, MBA

del Cañar. El 1\% de la región amazónica. El estudio refleja el 100\% de turismo interno nacional.

Se excluye en esta toma de datos el turismo extranjero $0 \%$. El hallazgo corresponde a la variable V1: Procedencia.

Para corroborar el hallazgo, acudimos a la fuente primaria, el libro de oro de visitas a la Virgen de la Nube en el Abuga, registro desde 29 de octubre de 2016 al 13 de enero del 2018. Siendo los hallazgos más relevantes los siguientes: Turismo Religioso Interno, $(84,95 \%)$. Turismo religioso extranjero, (15,05\%). La región sierra representa el $(75,27 \%)$. La costa el (8,60\%). La región amazónica representa el $(1,08 \%)$ de visitas en el periodo rastreado. Las ciudades que más incidencia de visitas tienen son Cuenca (33,33\%) y Azogues (18,28\%). Los países que más inciden en las visitas son: Estados Unidos (10,75\%) y España (2,15\%) (Véase tabla No. 1)

Lo relevante del hallazgo en la variable V2. Hábitos, es el carácter sociológico del turismo interno religioso. Así, el 73\% de las visitas lo hace en familia. En la línea de las inquietudes de orden gregario inclusive, se sitúa el $14 \%$ de turistas que se desplazan al destino turístico, con motivo de visita a la Virgen de la Nube, con amigos. Tan solo el 12\% lo hace de manera individual.

El tamaño del turismo, variable V3, denota la cantidad de personas por rangos que en función del hábito o variable V2, visitan el producto de turismo religioso. A excepción del 12\% que lo hace de manera individual. Entonces se pregunta: ¿Cuántas personas lo acompañan? En el rango de 0 a 5 personas, se encuentra que lo hace un 62\%. De 6 a 10, el 24\%. Más de 11 personas, el 14\%. A priori, son relevantes los datos, que bien podrían generar oportunidades para promocionar paquetes turísticos en el corredor: San Francisco-Señor de Flores- Virgen de la Nube en el Abuga, por ejemplo. 


\section{Impacto del turismo religioso en la ciudad de Azogues, Ecuador. Caso: Virgen}

de la Nube

Vol. 2, núm. Esp., (2018)

Ing. Florencio Iván García Álvarez, MBA; Ing. Juan Bautista Solis Muñoz, MBA; Ing. Xavier Augusto Mantilla Crespo, MBA

A nivel exploratorio y para tomar el pulso y dimensionar la viabilidad comercial de los emprendimientos turísticos, el equipo investigador definió la variable: Frecuencia de Turismo, V4. $\mathrm{Al}$ inicio de todos los años, lo hace un 41\%, lo que aparentemente equivale a una porción fidelizada al destino turístico en estudio. Por primera vez, un $21 \%$, este porcentaje equivale a la primera experiencia y comporta mayores estímulos de percepción. La opción varias veces al año, pesa un $27 \%$, porcentaje que debe ser considerado clave para impulsar acciones de emprendimiento turístico y mejorar los servicios del destino turístico, tanto del atractivo Santuario Franciscano como de la propia procesión de la Virgen de la Nube.

La variable canales de publicidad: V5, es relevante en la medida en que genera ideas de eficacia de los mensajes a través de los canales de comunicación. El 28\% se informa mediante avisos de la comunidad franciscana. Por radio, prensa y televisión, el 20\%. El 17\% de los mensajes llegan y tienen efecto mediante redes sociales. Mientras que el $32 \%$ se informa por todos los medios. Es marginal el efecto de las campañas del Ministerio de Turismo, que alcanza tan solo el 3\%. Sin embargo, rastreadas las redes sociales, sobre todo facebook se encuentra que el Mintur, con tres días de anticipación al 1 de enero registra publicaciones promocionales. Al parecer, la estrategia multimedial podría constituir una opción que potencie la información a los turistas potenciales alrededor del mundo, dado su comportamiento (32\%).

Entre los medios de transporte utilizados para los desplazamientos al destino turístico, registrados en la variable, V6, se halla: Vehículo particular, 43\%. Autobús de transporte público, alcanza un $42 \%$. A priori, los datos disparan la necesidad de una mayor intervención de los espacios públicos, vías, parqueaderos con la correspondiente señalética. El uso del servicio de 


\section{Impacto del turismo religioso en la ciudad de Azogues, Ecuador. Caso: Virgen \\ de la Nube}

Vol. 2, núm. Esp., (2018)

Ing. Florencio Iván García Álvarez, MBA; Ing. Juan Bautista Solis Muñoz, MBA; Ing. Xavier Augusto Mantilla Crespo, MBA

alquiler, taxi, camioneta es del $9 \%$. Apenas un $6 \%$ lo hace en transporte alternativo, lo que

evidencia, dependiendo de las distancias, la preferencia al transporte motorizado.

En cuanto a la señalética turística, variable V7, que recibe los datos de la pregunta: ¿Existe señalética turística en el trayecto hacia el Santurario Franciscano? revela que el 24\% señala que no existe señalética turística. Frente a un $70 \%$ que considera lo contrario. El hallazgo en función de la rigidez de la pregunta le llevó al equipo investigador a indagar vía grupos focales para validar el dato corroborando con la caracterización cualitativa de la investigación de campo.

¿Qué tiempo permanece en Azogues, por la visita a la Virgen de la Nube, en este 1 de enero? Interrogante que sustenta a la variable V8. Tiempo de permanencia en el destino. Si bien el 65\% responde que un día, los hallazgos siguientes: dos días el 19\%; tres días, el 9\%; y, más de tres días, el 7\% son principio pertinentes al desarrollo del producto turístico y la generación de nuevos servicios y rutas turísticas.

Es interesante el presente estudio a nivel exploratorio en la medida del desarrollo de la evidencia empírica y el comportamiento de los datos. Así la variable equipamiento de alojamiento: V9. Muestra que hoteles y hostales con un $6 \%$ y $7 \%$ respectivamente, frente al $24 \%$ de las casas de acogida y el $41 \%$ que se aloja en casas de familiares, aún podría implementar estrategias de mayor participación en el mercado.

El equipo investigador planteó la interrogante: ¿En su entorno ambiental y de limpieza cuál es su percepción de la ciudad de Azogues? La variable en estudio: Percepción del entorno natural y limpieza del destino, para esta pregunta dirigida a la ciudad de Azogues, V10. El 21\% lo percibe medianamente limpia. Tan solo un 4\% poco limpia, lo que estimula a la institución encargada por 


\section{Impacto del turismo religioso en la ciudad de Azogues, Ecuador. Caso: Virgen de la Nube}

Vol. 2, núm. Esp., (2018)

Ing. Florencio Iván García Álvarez, MBA; Ing. Juan Bautista Solis Muñoz, MBA; Ing. Xavier Augusto Mantilla Crespo, MBA

competencia constitucional y legal del aseo y ornato de la ciudad a intensificar las estrategias de

conservación de la imagen del destino. Al momento el 20\% lo considera muy limpia y el 55\% limpia. Esta percepción, a priori, debe ser aprovechada por las MIPYMES para potenciar el turismo receptivo.

V11. Asepsia y sabor de la gastronomía del destino, se pudo conocer anclada a la pregunta ¿La preparación y servicio de alimentos durante estos días con ocasión de la visita a la Virgen de la Nube, lo calificaría cómo? En efecto, el 47\% respondió que bueno. El 27\% dijo que muy bueno. Un 19\% lo calificó como excelente. Estudios derivados a mayor profundidad en la línea podrían discriminar las categorías en función de los factores determinantes de la calidad. Por ahora, a nivel exploratorio, los hallazgos no disponen de datos base comparable.

V12. Seguridad del destino turístico. La percepción de la muestra con relación a la variable seguridad es aceptable. El $41 \%$ percibe como buena la seguridad. Un $28 \%$ como muy buena. Un $23 \%$ como excelente. La estrategia apuntaría a mantener estos niveles de percepción en el turismo religioso porque abona de forma directa a potenciar el destino.

Se ha puesto singular interés, al definir la variable, V13. Consumo en dólares, para explorar en la ecuación de la renta que es igual al consumo, más el ahorro y más el pago de los impuestos, el comportamiento del gasto de consumo en relación al fenómeno estudiado. El rango de gasto entre 0 a 100 dólares norteamericanos, tiene un nivel del 82\% de incidencia. El intervalo de 101 a 500 dólares, representa el 17\% del estudio. Siendo marginal de 501 dólares en adelante con un 1\% de consumo. Estudios posteriores averiguarán la distribución del gasto por sectores de bienes y servicios. 


\section{Impacto del turismo religioso en la ciudad de Azogues, Ecuador. Caso: Virgen \\ de la Nube}

Vol. 2, núm. Esp., (2018)

Ing. Florencio Iván García Álvarez, MBA; Ing. Juan Bautista Solis Muñoz, MBA; Ing. Xavier Augusto Mantilla Crespo, MBA

V14. Potenciales atractivos encadenados. Pretende situar conexiones, entre intenciones de

visita y visitas reales, a los atractivos: La Virgen de la Nube en el Abuga, que obtiene un 54\%.

Seguidamente las aguas termales de Guapán, si bien no están concluidas todavía pero representan una inclinación de visita del 19\%. El complejo paisajístico de Cojitambo con un 12\%. Los museos de la ciudad de Azogues, representan un 7\% de intención de visita. De hecho, el rastreo del estado del arte, ha permito desarrollar aunque someramente la caracterización de los destinos turísticos estudiados en la presente variable.

En este momento se procede a la construcción de la matriz de variables clave en función de los factores de competitividad: Posicionamiento con un peso relativo del $30 \%$. Sostenibilidad con el $50 \%$. Rentabilidad con el $20 \%$. Se pondera bajo el criterio de alto impacto (3); mediana incidencia (1); y, no incide (0). Luego de las operaciones aritméticas se tiene en el rango de variables clave de alto impacto, es decir, a las que se tiene que atacar primero a: V3. Tamaño de turismo (3); V4. Frecuencia de Turismo (3); V5. Canales de Publicidad (3). (Véase gráficos 1, 2, y 3).

Las variables que le siguen en importancia de revisión son: V2. Hábitos de turismo $(2,4)$; V7. Señalética Turística $(2,4)$. V13. Consumo en dólares $(2,4)$. Las demás variables declaradas y medidas tendrán una atención gerencial de tercer momento. (Véase Tabla No. 2).

El equipo investigador decidió caracterizar testimonios de los turistas durante los cuatro días de levantamiento de información cerrada, para nutrir el marco de los hallazgos, corroborar en unos casos y en otros, inclusive contradecir los resultados, que en el nivel de discusión serán expuestos para la generación de hilos dinámicos de construcción académica, a más de la inmersión 


\section{Impacto del turismo religioso en la ciudad de Azogues, Ecuador. Caso: Virgen de la Nube}

Vol. 2, núm. Esp., (2018)

Ing. Florencio Iván García Álvarez, MBA; Ing. Juan Bautista Solis Muñoz, MBA; Ing. Xavier Augusto Mantilla Crespo, MBA

en estudios de similares inquietudes. Es decir, se desarrolla en adelante el enfoque cualitativo de

la presente investigación. Se quiso averiguar concretamente cual es la percepción de los turistas

nacionales y extranjeros sobre las variables independientes del producto de turismo religioso: La

Virgen de la Nube, venerada en el Santuario Franciscano de Azogues, con ocasión de la procesión

del 1 de enero de 2018. Los testimonios debidamente categorizados se describen a continuación:

Que no existe señalización en el Santuario se indica, ni en los atractivos turísticos de la ciudad. Se requiere información sobre los corredores turísticos con sus atractivos y destinos. Se menciona la necesidad de intervención vial en el acceso al cerro Abuga. No se dispone de servicios de parqueadero seguro o de existir el servicio no existe información mediante la difusión del plano de la ciudad. Se insiste que en El Cisne, existen espacios dedicados y seguros para estacionar los buses.

En la línea de los testimonios, se hace notar la inexistencia de personal que informe de manera personalizada a los turistas, sobre los servicios existentes, opciones de alimentación, hospedaje, lugares turísticos cercanos, variedad de gastronomía, actividades culturales nocturnas. Se menciona que es la comunidad franciscana la única que sostiene e informa sobre la procesión del 1 de enero en Azogues. Si bien existen los ejercicios espirituales, se menciona que no se dispone de guías turísticos profesionales que cuente la historia de la Virgen y otros episodios como la justificación de la celebración del 1 de enero y la presencia de la Virgen de la Nube en Azogues, sabiendo que la aparición fue en Quito, así también se anhela conocer la historia de la ciudad, leyendas, tradiciones, fechas memorables, barrios tradicionales. Este año se menciona como hecho que llama la atención el cambio de lugar para la celebración de la misa y procesión, sobre todo, se enfatiza en la falta de información previa. 


\section{Impacto del turismo religioso en la ciudad de Azogues, Ecuador. Caso: Virgen \\ de la Nube}

Vol. 2, núm. Esp., (2018)

Ing. Florencio Iván García Álvarez, MBA; Ing. Juan Bautista Solis Muñoz, MBA; Ing. Xavier Augusto Mantilla Crespo, MBA

Según los testimonios, predomina la fe y la tradición como motivos potentes de la visita, en el eje de la fe y la religiosidad popular. Sin embargo, hay interés por el turismo interno y la visita a atractivos como Hatun Pamba, Cojitambo, Abuga. Los turistas se quedaban un solo día, ya que solo visitaban a la virgen en particular, sin embargo, otras personas se quedaban para visitar otros lugares como, Hatun pamba, Cojitambo, el Abuga. Como que el turismo religioso sostenido desde la historia mediante seria investigación de fuentes primarias, aún no está explotado, ni contado.

Mediante grupos focales, se levanta información testimonial de los actores de la economía popular y solidaria. Para Jorge Tenecota, del Romántico Restaurante, en estos dos últimos años ha afectado la eucaristía en el estadio municipal. Refiere que no se está aprovechando al 100\% el flujo turístico que visita a la Virgen de la Nube. Agregan este mismo sector, Martha Luna, Mélida López y Ana Bonete, que además no existe control al punto que los domicilios se convierten en restaurantes y casas de venta de comida. No existen guías turísticos ni señalética turística sobre todo hacia la Virgen de la Nube en el Abuga. Desde el sector del transporte turístico, Marco Navas y Washinton Peñafiel, manifiestan que el 1 de enero se consideran meros espectadores, los feligreses usan vehículos particulares y transportación pública, en este último caso, cuentan con salvo conductos de la Agencia Nacional de Tránsito, muy a pesar de ser turismo religioso que no están autorizados en los permisos de operación. En opinión de Esperanza Regalado, de hostal Peleusí, los turistas se alojan los días previos, pero el uno de enero parten a sus ciudades de procedencia. No existe programación cultural de ninguna entidad para los feligreses que visitan a la Virgen de la Nube el 1 de enero. Lo hallado se contrapone a lo que si realiza el gobierno autónomo descentralizado de la ciudad de Loja, en el caso de la Virgen del Cisne, cuenta con la 


\section{Impacto del turismo religioso en la ciudad de Azogues, Ecuador. Caso: Virgen}

de la Nube

Vol. 2, núm. Esp., (2018)

Ing. Florencio Iván García Álvarez, MBA; Ing. Juan Bautista Solis Muñoz, MBA; Ing. Xavier Augusto Mantilla Crespo, MBA

guía turística de la Región Sur del Ecuador. Bajo el eslogan Loja, linda como su gente, promociona

el turismo religioso, aunque el producto Virgen del Cisne está en el imaginario de la feligresía que entre 25 mil y 30 mil personas visitan año tras año Loja y trasmiten de generación en generación la devoción a la advocación del Cisne. El Municipio de Loja, desarrolla con motivo de las festividades de la Virgen del Cisne una programación cultural dedicada a los feligreses, usando los espacios públicos existentes en la ciudad.

\section{Discusión.}

La investigación claramente plantea una mirada desde la fe y la historia del turismo religioso como fuente de oportunidades. Aparentemente los países de América no pasaron en la concepción espacio tiempo, la edad media, que sin embargo, en Europa gesta el 90\% de la iconografía contenida en fichas con ubicación, tema, serie, análisis compositivo. América se quedó en el rezo de la fe y la religiosidad popular. No se ha investigado por ejemplo ¿Qué elementos religiosos tenemos dentro del arte profano que todavía perviven? o los signos y símbolos utilizados como catequesis visual para evangelizar. Tomando como caso de estudio a la Virgen de la Nube, venerada en el Santuario Franciscano de la ciudad de Azogues, con información levantada en forma previa y durante la procesión del 1 de enero de 2018, donde los hilos fuertes de discusión en función de los hallazgos cuantitativos y cualitativos se centran en el real aprovechamiento de las oportunidades para desarrollar el destino y generar encadenamientos de servicios a lo largo de los corredores turísticos religiosos: San Francisco- Señor de Flores- Virgen de la Nube del Abuga; o, los no religiosos como atractivos museográficos y etnográficos existentes en la ciudad de Azogues. Las potencialidades de las aguas termales de Guapán. El mirador paisajístico y de 


\section{Impacto del turismo religioso en la ciudad de Azogues, Ecuador. Caso: Virgen \\ de la Nube}

Vol. 2, núm. Esp., (2018)

Ing. Florencio Iván García Álvarez, MBA; Ing. Juan Bautista Solis Muñoz, MBA; Ing. Xavier Augusto Mantilla Crespo, MBA

turismo de escalada en Cojitambo. Tomando en cuenta la devoción de la Virgen de la Nube como

Madre del Ecuatoriano Ausente en Estados Unidos y Canadá, relacionado al concepto espiritual de los migrantes y los flujos de remesas enviados por ellos a sus familias particularmente en el Austro del país. Se ha rastreado el Impacto del factor religioso de la comunidad ecuatoriana en Madrid. El caso de la Virgen del Cisne, que tiene como objetivo principal analizar el fenómeno social que se produce con una tradición de Ecuador (...) en la parroquia de San Lorenzo de Lavapiés en Madrid, España. Se arriba al análisis sobre las raíces comunes y tradiciones procedentes de España con las semejanzas que existen en cuanto a la veneración a la Virgen María. Aparentemente, el estudio que nos pertenece se fundamenta en medir variables consideradas clave, de mediano y bajo impacto así como caracterizar las percepciones de los turistas visitantes. Los hitos en común de los estudios comparados se leen en la influencia que ejerce aún la iglesia católica en la generación y sostenimiento de manifestaciones espirituales de fe y tradición mariana, desprendido del enfoque cualitativo de la presente investigación.

El turismo religioso desde la mirada del impacto económico es una tarea pendiente. Lourdes y Fátima, los focos de peregrinación más grandes de Europa, en ella, toda la población involucrada en el área de influencia ha progresado, ha prosperado como producto del orden, todo está pensado y preparado para que la gente tenga todo lo que necesita. En la línea de crear orden para el destino turístico en análisis, los insumos para la discusión desde el enfoque cuantitativo, son las variables clave de alto y mediano impacto en los llamados factores de competitividad: Tamaño de turismo; frecuencia de turismo, canales de publicidad, con un puntaje ponderado de 3. Entre tanto, hábitos de turismo, señalética turística y consumo en dólares tienen 2,4 de puntaje ponderado. Para esto, no existe evidencia empírica que sitúe estudios cuantitativos extrapolables, 


\section{Impacto del turismo religioso en la ciudad de Azogues, Ecuador. Caso: Virgen}

de la Nube

Vol. 2, núm. Esp., (2018)

Ing. Florencio Iván García Álvarez, MBA; Ing. Juan Bautista Solis Muñoz, MBA; Ing. Xavier Augusto Mantilla Crespo, MBA

sino más bien, apunta a mover los cimientos de la política pública en los diversos niveles de la gestión de gobierno, mediante la implementación de planes de acción consolidados a las prioridades establecidas en la rigurosidad metodológica. El hilo per se de discusión se fundamenta en la pregunta: ¿Es posible entonces y qué tiempo, reversar las debilidades del destino turístico y potenciar el aprovechamiento de las oportunidades que genera el turismo religioso en Azogues?

\section{Conclusión.}

No existe señalización en el interior del Santuario y en las áreas de influencia que conectan los diferentes equipamientos, tampoco en los atractivos turísticos de la ciudad, se requiere información hipertextual impresa y digital;

No se dispone de servicios de parqueadero seguro o de existir el servicio no existe información mediante la difusión del plano de la ciudad. Se necesitan espacios dedicados y seguros para estacionar los buses;

Inexistencia de guías que informen de manera personalizada a los turistas, sobre los servicios existentes, opciones de alimentación, hospedaje, lugares turísticos cercanos, variedad de gastronomía, actividades culturales nocturnas. Se requiere de guías turísticos profesionales que cuenten la historia de la Virgen y otros episodios como la justificación de la celebración del 1 de enero y la presencia de la Virgen de la Nube en Azogues. Se anhela conocer la historia de la ciudad, leyendas, tradiciones, fechas memorables, barrios tradicionales, en base a investigación previa hecha sobre fuentes primarias; 


\section{Impacto del turismo religioso en la ciudad de Azogues, Ecuador. Caso: Virgen \\ de la Nube}

Vol. 2, núm. Esp., (2018)

Ing. Florencio Iván García Álvarez, MBA; Ing. Juan Bautista Solis Muñoz, MBA; Ing. Xavier Augusto Mantilla Crespo, MBA

Este año, llama la atención, el cambio de lugar para la celebración de la misa y procesión,

sobre todo, se enfatiza en la falta de información previa, como ocurrió también en la ciudad de Loja, la Virgen del Cisne no llegó a la Catedral, sino al templo de San Sebastián, generando confusión en la feligresía;

Predomina la fe y la tradición como motivos potentes de la visita. Hay interés por el turismo interno y la visita a atractivos como Hatun Pamba, Cojitambo, Abuga. Para ello, es básico constar como prioridad en la Agenda Zonal, alineada al Plan Nacional del Buen Vivir Toda una Vida que asegure la inversión de recursos sociales en proyectos como el teleférico del Abuga;

El tamaño del turismo, variable V3. En el rango de 0 a 5 personas, se encuentra que lo hace un $62 \%$. De 6 a 10, el 24\%. Más de 11 personas, el 14\%. A priori, son relevantes los datos, que bien podrían generar oportunidades para promocionar paquetes turísticos en el corredor: San Francisco-Señor de Flores- Virgen de la Nube en el Abuga, por ejemplo.

Frecuencia de Turismo, V4. Al inicio de todos los años, lo hace un $41 \%$, lo que aparentemente equivale a una porción fidelizada al destino turístico en estudio. Por primera vez, un $21 \%$, este porcentaje equivale a la primera experiencia y comporta mayores estímulos de percepción. La opción varias veces al año, pesa un 27\%, porcentaje que debe ser considerado clave para impulsar acciones de emprendimiento turístico y mejorar los servicios del destino turístico, tanto del atractivo Santuario Franciscano como de la propia procesión de la Virgen de la Nube.

La variable canales de publicidad: V5, es relevante en la medida en que genera ideas de eficacia de los mensajes a través de los canales de comunicación. El 28\% se informa mediante avisos de la comunidad franciscana. Por radio, prensa y televisión, el 20\%. El 17\% de los mensajes 


\section{Impacto del turismo religioso en la ciudad de Azogues, Ecuador. Caso: Virgen}

de la Nube

Vol. 2, núm. Esp., (2018)

Ing. Florencio Iván García Álvarez, MBA; Ing. Juan Bautista Solis Muñoz, MBA; Ing. Xavier Augusto Mantilla Crespo, MBA

llegan y tienen efecto mediante redes sociales. Mientras que el $32 \%$ se informa por todos los

medios. Es marginal el efecto de las campañas del Ministerio de Turismo, que alcanza tan solo el

3\%. Al parecer, la estrategia multimedial podría constituir una opción que potencie la información

a los turistas potenciales alrededor del mundo, dado su comportamiento (32\%);

En la Agenda Zonal para la Región de Planificación 6, debe contemplar en el bio corredor del Sur, el turismo religioso como prioridad de desarrollo sostenido;

La formulación de los planes de acción derivados de un plan estratégico y táctico que sostenga a los factores de competitividad, deben estar en manos de la Gerencia de Gestión Turística, que eventualmente llevaría el nombre de Virgen de la Nube, para desarrollar una prospectiva a largo aliento y aprovechar de mejor manera las potencialidades del turismo religioso en Azogues;

La Comunidad Franciscana debe crear una Gerencia de Gestión Turística, adscrita a la máxima Autoridad Franciscana en Azogues, con autonomía responsable para que gestione integralmente el producto turístico y establezca relaciones de cooperación eficaces con los sectores público y comunitario, en los dos anclajes del turismo religioso;

Se debe hacer estudios iconográficos integrales, basados en fuentes primarias o documentales sobre la línea del turismo religioso;

En base a la investigación a profundidad, elaborar las cartillas didácticas para educación escolar y uso de los guías turísticos. 


\section{Impacto del turismo religioso en la ciudad de Azogues, Ecuador. Caso: Virgen}

de la Nube

Vol. 2, núm. Esp., (2018)

Ing. Florencio Iván García Álvarez, MBA; Ing. Juan Bautista Solis Muñoz, MBA; Ing. Xavier Augusto Mantilla Crespo, MBA

Anexos.

Tabla No 2: Procedencia de feligreses a la Virgen de la Nube del Abuga

\begin{tabular}{|l|r|l|r|}
\hline \multicolumn{1}{|c|}{ ECUADOR } & \% de visitas & EXTRANJERO & \% de visitas \\
\hline Azuay & $41,94 \%$ & España & $2,15 \%$ \\
\hline Cañar & $26,88 \%$ & Canadá & $1,08 \%$ \\
\hline Guayas & $5,38 \%$ & Estados Unidos & $10,75 \%$ \\
\hline Manabi & $3,23 \%$ & Holanda & $1,08 \%$ \\
\hline Morona Santiago & $1,08 \%$ & & \\
\hline Pichincha & $2,15 \%$ & & \\
\hline Chimborazo & $2,15 \%$ & & \\
\hline Loja & $2,15 \%$ & & $\mathbf{1 5 , 0 5 \%}$ \\
\hline TOTAL & $\mathbf{8 4 , 9 5 \%}$ & TOTAL & \\
\hline
\end{tabular}

Fuente: García, Solis, Mantilla. Ecuador, 2018

Tabla No 2: Variables clave en función de los factores de competitividad

Impacto del turismo religioso en la ciudad de Azogues, Ecuador. Caso: Virgen de la Nube

\begin{tabular}{|c|c|c|c|c|c|c|c|}
\hline Variables & \multicolumn{7}{|c|}{ Factores de Competividad } \\
\hline & \multicolumn{2}{|c|}{ Posicionamiento $(0,30)$} & \multicolumn{2}{|c|}{ Sosteniblidad $(0,50)$} & \multicolumn{2}{|c|}{ Rentabilidad $(0,20)$} & \multirow{2}{*}{\begin{tabular}{r|} 
Total \\
0,8
\end{tabular}} \\
\hline V1. Procedencia & 1 & 0,3 & 1 & 0,5 & 0 & 0 & \\
\hline V2. Hábitos de Turismo & 1 & 0,3 & 3 & 1,5 & 3 & 0,6 & 2,4 \\
\hline V3. Tamaño de turismo & 3 & 0,9 & 3 & 1,5 & 3 & 0,6 & 3 \\
\hline V4. Frecuencia de Turismo & 3 & 0,9 & 3 & 1,5 & 3 & 0,6 & 3 \\
\hline V5. Canales de Publicidad & 3 & 0,9 & 3 & 1,5 & 3 & 0,6 & 3 \\
\hline V6. Medios de Transporte & 0 & 0 & 1 & 0,5 & 0 & 0 & 0,5 \\
\hline V7. Señalética Turística & 1 & 0,3 & 3 & 1,5 & 3 & 0,6 & 2,4 \\
\hline V8. Tiempo de permanencia en el destino & 0 & 0 & 3 & 1,5 & 3 & 0,6 & 2,1 \\
\hline V9. Equipamientos de alojamiento & 0 & 0 & 3 & 1,5 & 3 & 0,6 & 2,1 \\
\hline $\begin{array}{l}\text { V10. Percepción del entorno natural y limpieza } \\
\text { del destino }\end{array}$ & 1 & 0,3 & 3 & 1,5 & 1 & 0,2 & 2 \\
\hline $\begin{array}{l}\text { V11. Asepsia y sabor de la gastronomía del } \\
\text { destino }\end{array}$ & 1 & 0,3 & 3 & 1,5 & 0 & 0 & 1,8 \\
\hline V12. Seguridad del destino turístico & 1 & 0,3 & 3 & 1,5 & 1 & 0,2 & 2 \\
\hline V13. Consumo en dólares & 1 & 0,3 & 3 & 1,5 & 3 & 0,6 & 2,4 \\
\hline V14. Potenciales atractivos encadenados & 0 & 0 & 1 & 0,5 & 3 & 0,6 & 1,1 \\
\hline
\end{tabular}

Fuente: García, Solis, Mantilla. Ecuador, 2018 


\section{Impacto del turismo religioso en la ciudad de Azogues, Ecuador. Caso: Virgen}

de la Nube

Vol. 2, núm. Esp., (2018)

Ing. Florencio Iván García Álvarez, MBA; Ing. Juan Bautista Solis Muñoz, MBA; Ing. Xavier Augusto Mantilla Crespo, MBA

Gráfico No. 1: Variable Tamaño de turismo

\section{3. ¿Cuántas personas le acompañan?}

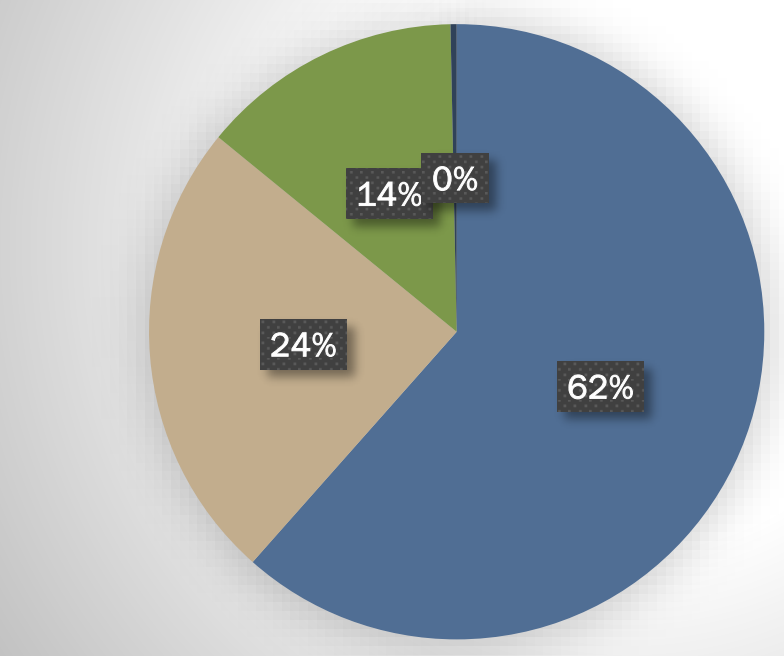

De 0-5

DE 6-10

- Más de 11 personas

- N/C

Fuente: García, Solis, Mantilla. Ecuador, 2018

Gráfico No. 2. Variable Frecuencia de turismo

\section{4. ¿La visita a la Virgen de la Nube, lo} hace?

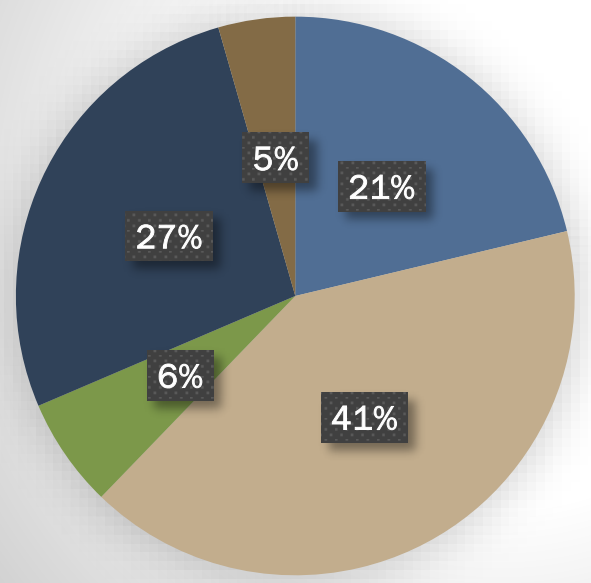

- Por primera vez

A inicio de todos los años

Dos veces al año

- Varias veces al año

- Otro

Fuente: García, Solis, Mantilla. Ecuador, 2018 


\section{Impacto del turismo religioso en la ciudad de Azogues, Ecuador. Caso: Virgen}

de la Nube

Vol. 2, núm. Esp., (2018) Ing. Florencio Iván García Álvarez, MBA; Ing. Juan Bautista Solis Muñoz, MBA; Ing. Xavier Augusto Mantilla Crespo, MBA

Gráfico No. 3: Variable Canales de Publicidad

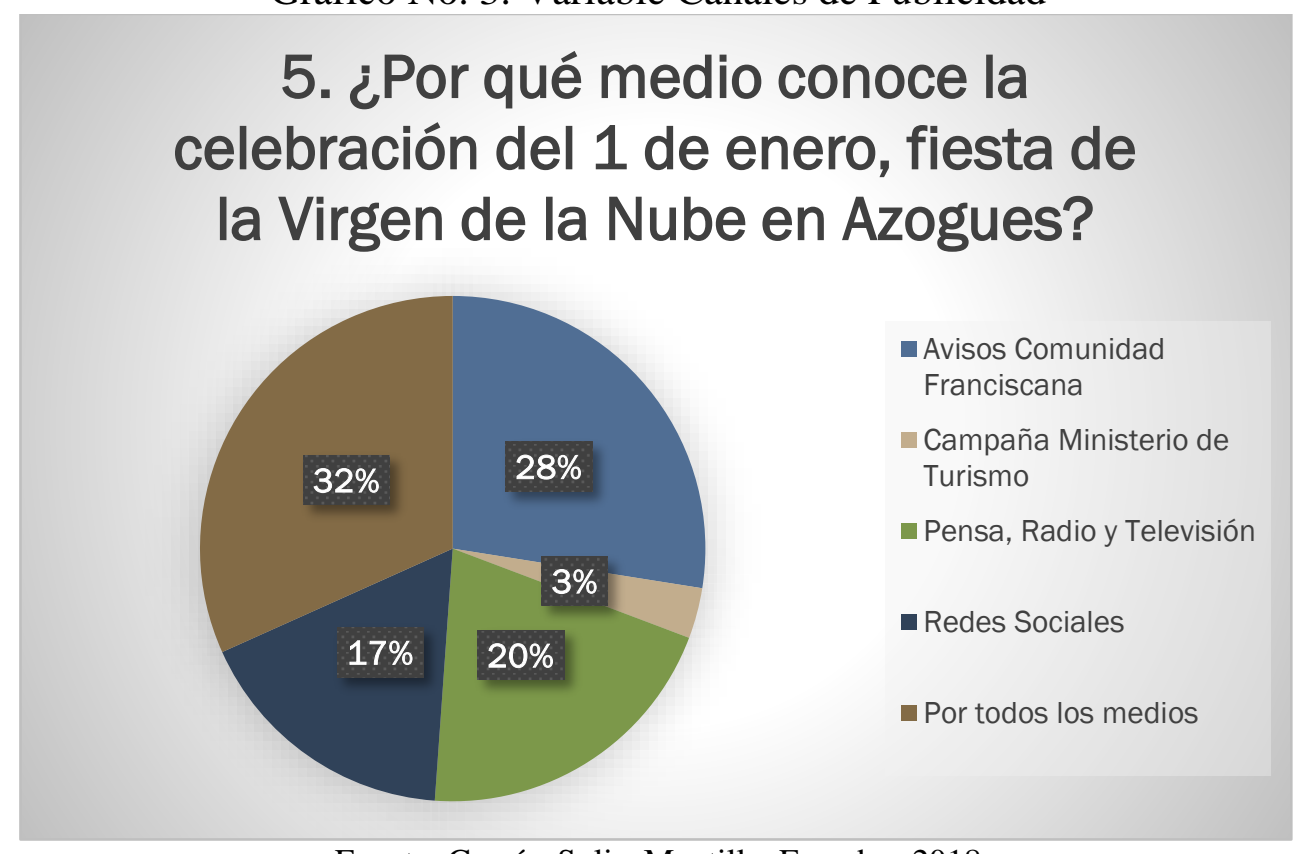

Fuente: García, Solis, Mantilla. Ecuador, 2018 
Impacto del turismo religioso en la ciudad de Azogues, Ecuador. Caso: Virgen de la Nube

Vol. 2, núm. Esp., (2018)

Ing. Florencio Iván García Álvarez, MBA; Ing. Juan Bautista Solis Muñoz, MBA; Ing. Xavier Augusto Mantilla Crespo, MBA

Referencias.

Arias, J. (2018). Nuestra Señora del Cisne. Editorial Hernández, Cuenca - Ecuador;

Armijos, M. (2015). Plan de concientización ambiental en la Romería del Quinche en la Provincia de Pichincha. Publicación del Instituto Tecnológico Cordillera;

Carrasco, F. (2016). Azogues y el Cañar Turísticos. Editado por la Municipalidad de AzoguesEcuador;

CulturisArte, (2017). La Virgen de la Nube en la cima del Abuga. Azogues- Ecuador;

Domínguez, C. (2006). Crónicas y Documentos de la Fundación del Convento Franciscano y del Santuario de la Santísima Virgen de la Nube 1912- 2003. Editorial Hernández. Cuenca Ecuador;

Ecuador Franciscano, (1943). Escultura del Santuario. Págs: 167, 168 y 231;

Eljuri, G. (2005). La Virgen de la Nube en Manhattan. Religiosidad Popular más allá de la frontera.

Guía Turística de la Región Sur del Ecuador, (2008). Región 7: El Oro, Loja y Zamora Chinchipe. Loja - Ecuador;

La Ruta de la Cascarrilla. Archivo Histórico de Loja (2018). Loja - Ecuador;

Libro de Oro, (2016). Registro cronológico de visitas a la Virgen de la Nube en el cerro Abuga. Azogues- Ecuador;

López, A. (1583). Relaciones de la Audiencia de Quito;

Matovelle, J. (1900). Nuestra Señora de la Nube. Imprenta del Clero. Cuenca - Ecuador (págs. 167179);

Orellana, J. (1948). Resumen Histórico del Ecuador. Biblioteca Nacional del Ecuador: Eugenio Espejo. Quito- Ecuador;

Peñafiel, R. (2008). Diagnóstico del II Festival del Maíz de Cojitambo. Unidad Académica de Ciencias Administrativas y Empresariales. UCACUE- Sede Azogues;

Plan Nacional del Buen Vivir, (2013- 2017)

Plan Nacional del Buen Vivir Toda una Vida (2017-2021)

Plan de Desarrollo Urbano de Azogues (1990). Azogues - Ecuador; 


\section{Impacto del turismo religioso en la ciudad de Azogues, Ecuador. Caso: Virgen de la Nube}

Vol. 2, núm. Esp., (2018)

Ing. Florencio Iván García Álvarez, MBA; Ing. Juan Bautista Solis Muñoz, MBA; Ing. Xavier Augusto Mantilla Crespo, MBA

Planificación para las provincias de Azuay, Cañar y Morona Santiago. Agenda Zonal (20132017). Secretaría Nacional de Planificación y Desarrollo Senplades;

Pérez, S. (2007). Impacto del Factor Religioso en la Comunidad Ecuatoriana en Madrid. El caso de la Virgen del Cisne. Revista Dilemas Contemporáneos: Educación, Política y Valores. Número 2. ISSN: 2007-7890;

Ramírez, G. (1998). Cañar: Provincia Emérita. Azogues- Ecuador;

Revista, (2012). Un siglo de fe y prodigios. Edición conmemorativa centenaria. Azogues- Ecuador;

Toledo, M. (2007). Fiestas Populares una Alternativa del Turismo Cultural en la Provincia del Cañar. Universidad del Azuay. Cuenca- Ecuador;

Tobay, C. (2008). Ruta religiosa para la ciudad de Loja. Universidad del Azuay. Cuenca - Ecuador;

Ulloa, E. (2009). Elaboración de un Paquete de Turismo Religioso en las provincias de Azuay y Cañar. Universidad del Azuay. Cuenca - Ecuador;

Vicuña, J. (2018). Libro de obra de fiscalización del proyecto turístico de aguas termales de Guapán. GPC. Azogues- Ecuador. 